\title{
A Generalized Constant Elasticity of Substitution Production Function Model and Its Application
}

\author{
Maolin CHENG \\ School of Mathematics and Physics, Suzhou University of Science and Technology, Suzhou 215009, \\ China \\ E-mail: ustscml@163.com
}

\begin{abstract}
The constant elasticity of substitution production function describes the relationship between production results and production factors in the technological production process. The common production factors include capital and labor. In order to comprehensively reflect the input-output relationship, this paper generalizes the model and adds factors including energy, consumption, and import and export. With respect to estimating the parameters of the model, the paper proposes a high-precision and high-speed nonlinear regression method. The constant elasticity of substitution production function model is mainly used to calculate the contribution rates of economic growth factors, and this paper proposes a scientific and reliable calculating method. The final section of the paper proposes an empirical analysis of the contribution rates of Chinese economic growth factors.
\end{abstract}

Keywords production function; economic growth; contribution rate; nonlinear regression; empirical analysis

\section{Introduction}

In some cases, research on economic growth theory can be converted into research on the production function that is the mathematical expression of the dependent relationship between production factor inputs and outputs. Various production functions have been proposed by economists, the function that has had the greatest influence was proposed by Professor Solow of Massachusetts Institute of Technology, a past winner of a Nobel Prize in Economics. The production function is as follows

$$
Y=A f(K, L),
$$

where $Y$ represents output, $K$ represents capital input, $L$ represents labor input and $A$ represents technological level.

In production functions, the constant elasticity of substitution production function is an important formula. It has many forms, and the form commonly adopted is

$$
Y=A\left(\delta_{1} K^{-\rho}+\delta_{2} L^{-\rho}\right)^{-\frac{\gamma}{\rho}} .
$$

In the paper, this form is generalized as ${ }^{[1-3]}$

$$
Y=A_{0} \mathrm{e}^{\lambda t}\left(\delta_{1} K^{-\rho}+\delta_{2} L^{-\rho}+\delta_{3} E^{-\rho}+\delta_{4} C^{-\rho}+\delta_{5} I^{-\rho}\right)^{-\frac{\gamma}{\rho}},
$$

Received November 7, 2015, accepted January 6, 2016

Supported by National Natural Science Foundation of China (11401418) 
where $Y$ is output, $K$ is capital input, $L$ is labor input, $E$ is energy input, $C$ is consumption and $I$ is import and export. $A(t)=A_{0} \mathrm{e}^{\lambda t}$ represents the level of technological progress; $\delta_{1}, \delta_{2}, \delta_{3}, \delta_{4}, \delta_{5}$ represent the technically intensive degree of capital, labor, energy, consumption and import and export, respectively; $\gamma$ represents the function's homogeneous order or return to scale and $\gamma>0 ; \rho$ is the substitution parameter and $\rho \geq-1$; and $\lambda$ is the rate of technological progress. $A_{0}, \lambda, \delta_{1}, \delta_{2}, \delta_{3}, \delta_{4}, \delta_{5}, \rho, \gamma$ are all parameters to be estimated.

The constant elasticity of substitution production function describes the relationship between production results and production factors in the technological production process. If the general nonlinear regression method ${ }^{[4-6]}$ is used to estimate the parameters of the model, the results may be easily affected by the initial value, resulting in a low convergence rate and a lack of precision. Therefore, an improved nonlinear regression method ${ }^{[7-11]}$ is proposed in this paper. As to its application, the constant elasticity of substitution production function is mainly used to calculate the contribution rates of economic growth factors, and this paper proposes a scientific and reliable calculation method ${ }^{[12-17]}$. In the final section of this paper, an empirical analysis of the contribution rates of Chinese economic growth factors is given.

\section{Parameter Estimation of Constant Elasticity of Substitution Pro- duction Function Model}

The constant elasticity of substitution production function model is expressed as

$$
Y=f(X, \eta)+\varepsilon
$$

where $X=(K, L, E, C, I), \eta=\left(A_{0}, \lambda, \delta_{1}, \rho, \delta_{2}, \delta_{3}, \delta_{4}, \delta_{5}, \gamma\right)$, and $\varepsilon$ is the error margin in the fitting of the model.

Let $\sum_{i=1}^{n} \varepsilon_{i}^{2}=G(\eta)=\sum_{t=1}^{n}\left[Y_{t}-f\left(X_{t}, \eta\right)\right]^{2}$ and let it be set to the minimum.

The model solution is a nonlinear optimization problem that can be solved with the usual nonlinear optimization algorithms, but the objective function is relatively complicated because its gradient and Hessian matrix expressions are more complicated. This means that results will be more easily affected by the initial value, it is difficult to make them converge. Even if it is convergence, the rate of convergence is slow and the precision is poor. Therefore, in this paper, a mixed algorithm with the first-order derivative alone is established for model solving. Combining the quasi-Newton method and the trust region method, the mixed algorithm has the quasi-Newton method's advantage of global convergence with a quick rate of convergence and also the trust region method's advantage of high precision under certain conditions.

The gradient of the objective function $G(\eta)$ is expressed as

$$
\begin{aligned}
g(\eta) & =\left(\frac{\partial G}{\partial A_{0}}, \frac{\partial G}{\partial \lambda}, \frac{\partial G}{\partial \delta_{1}}, \frac{\partial G}{\partial \rho}, \frac{\partial G}{\partial \delta_{2}}, \frac{\partial G}{\partial \delta_{3}}, \frac{\partial G}{\partial \delta_{4}}, \frac{\partial G}{\partial \delta_{5}}, \frac{\partial G}{\partial \gamma}\right)^{\mathrm{T}} \\
& =\left(g_{1}\left(\eta_{1}\right), g_{2}\left(\eta_{2}\right), \cdots, g_{9}\left(\eta_{9}\right)\right)^{\mathrm{T}} .
\end{aligned}
$$

Let $g^{(k)}=g\left(\eta^{(k)}\right)$, and the algorithm steps are as follows:

1) Initialization. Assume the following conditions $\eta^{(0)} \in R^{m+r+1}, \rho^{(0)}>0,0<\sigma<\gamma<1 / 2$, $(m+r+1)$-order positive definite matrix $B^{(0)}, \Delta^{(0)}>0,0<d_{1}<1<d_{2}, \tilde{\Delta}>0$, and make $A^{(0)}=B^{(0)}+\rho^{(0)} I, \theta^{(0)}=g^{(0)}, k \Leftarrow 0 ;$ 
2) Execute the quasi-Newton step. Solve the equation set $A^{(k)} \delta=-g^{(k)}$ and the solution is $\delta^{(k)}$. If

$$
G\left(\eta^{(k)}+\delta^{(k)}\right)-G\left(\eta^{(k)}\right) \leq \sigma g^{(k)^{\mathrm{T}}} \delta^{(k)},
$$

let $\eta^{(k+1)}=\eta^{(k)}+\delta^{(k)}$,

$$
\gamma^{(k)}=\frac{G\left(\eta^{(k)}+\Delta^{(k)}\right)-G\left(\eta^{(k)}\right)}{g^{(k)^{\mathrm{T}}} \delta^{(k)}}
$$

Turn to 3); otherwise, turn to 2).

3) Execute the trust region step. Solve the following strictly convex constrained quadratic programming

$$
\begin{aligned}
\min & g^{(k)^{\mathrm{T}}} \delta+\frac{1}{2} \delta^{\mathrm{T}} A^{(k)} \delta \\
\text { s.t } & \|\delta\| \leq \Delta^{(k)},
\end{aligned}
$$

and find solution $\delta^{(k)}$. If

$$
G\left(\eta^{(k)}+\delta^{(k)}\right)-G\left(\eta^{(k)}\right) \leq \sigma g^{(k)^{\mathrm{T}}} \delta^{(k)},
$$

make $\eta^{(k+1)}=\eta^{(k)}+\delta^{(k)}$,

$$
\gamma^{(k)}=\frac{G\left(\eta^{(k)}+\delta^{(k)}\right)-G\left(\eta^{(k)}\right)}{g^{(k)^{\mathrm{T}}} \delta^{(k)}},
$$

and turn to 3); otherwise, let $\delta^{(k)}=d_{1} \Delta^{(k)}$ and turn to 2$)$.

4) Quasi-Newton modification step. If $\left\|\delta^{(k)}\right\|<\varepsilon$, stop; otherwise, make

$$
B^{(k+1)}=B^{(k)}-\frac{1}{\delta^{(k)^{\mathrm{T}}} B^{(k)} \delta^{(k)}} B^{(k)} \delta^{(k)} B^{(k)^{\mathrm{T}}} B^{(k)}+\frac{1}{\alpha^{\mathrm{T}} \delta^{(k)}} \alpha \alpha^{\mathrm{T}},
$$

where $\alpha=g^{(k+1)}-g^{(k)}$.

Let

$$
\Delta^{(k+1)}= \begin{cases}\Delta^{(k)}, & \text { if } \gamma^{(k)} \in[\sigma, \gamma] \\ \min \left(\tilde{\Delta}, d_{2} \Delta^{(k)}\right), & \text { else. }\end{cases}
$$

Calculate $\xi=g^{(k+1)}$.

Let

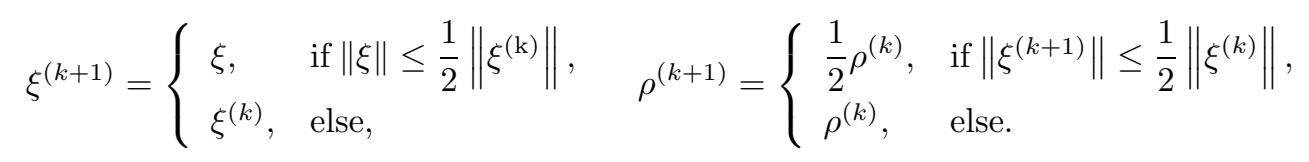

Let $k \Leftarrow k+1$,and then turn to 1 ).

\section{Calculation Method for the Contribution Rates of Economic Growth Factors}

The production function is denoted as $Y=f\left(X_{1}, X_{2}, \cdots, X_{m}\right)$. Assume factor $X_{i}$ changes over time, and each factor's values at $n$ points are known. In other words, $n$ points in $m$ dimensional space can be expressed as

$$
M_{1}\left(X_{1}^{1}, X_{2}^{1}, \cdots, X_{m}^{1}\right)
$$




$$
\begin{gathered}
M_{2}\left(X_{1}^{2}, X_{2}^{2}, \cdots, X_{m}^{2}\right), \\
\vdots \\
M_{n}\left(X_{1}^{n}, X_{2}^{n}, \cdots, X_{m}^{n}\right),
\end{gathered}
$$

where $X_{j}^{i}$ is the value of factor $j$ at point $i$, and points $M_{1}$ and $M_{n}$ are the values of factors at the starting and ending points of the corresponding analysis period.

Then, assume:

1) Production function $Y=f\left(X_{1}, X_{2}, \cdots, X_{m}\right)$ is differentiable;

2) $L^{(i)}$ is the curve connecting $M_{i}$ with $M_{i+1}(i=1,2, \cdots, n-1)$.

Let $\Delta Y_{i j}$ be the influence of the $j$ factor on the output change in period $i$, and then

$$
\Delta Y_{i j}=\int_{L^{(i)}} \frac{\partial f\left(X_{1}, X_{2}, \cdots, X_{m}\right)}{\partial X_{j}} \mathrm{~d} X_{j}, \quad j=1,2, \cdots, m
$$

Calculate all integrals to obtain the following matrix

$$
\left(\begin{array}{cccccc}
\Delta Y_{11} & \Delta Y_{12} & \ldots & \Delta Y_{1 j} & \ldots & \Delta Y_{1 m} \\
\Delta Y_{21} & \Delta Y_{22} & \ldots & \Delta Y_{2 j} & \ldots & \Delta Y_{2 m} \\
\ldots & \ldots & \ldots & \ldots & \ldots & \ldots \\
\Delta Y_{i 1} & \Delta Y_{i 2} & \ldots & \Delta Y_{i j} & \ldots & \Delta Y_{i m} \\
\ldots & \ldots & \ldots & \ldots & \ldots & \ldots \\
\Delta Y_{(n-1) 1} & \Delta Y_{(n-1) 2} & \ldots & \Delta Y_{(n-1) j} & \ldots & \Delta Y_{(n-1) m}
\end{array}\right)
$$

where factor $\Delta Y_{i j}$ shows the influence of the $j$ factor on the gross change in period $i$.

Calculate the value of $\Delta Y_{i j}$ by totaling rows to obtain the following equation determinant

$$
\begin{aligned}
& \left(\Delta Y_{1}, \Delta Y_{2}, \cdots, \Delta Y_{j}, \cdots, \Delta Y_{m}\right) \\
= & \left(\sum_{i=1}^{n-1} \Delta Y_{i 1}, \sum_{i=1}^{n-1} \Delta Y_{i 2}, \cdots, \sum_{i=1}^{n-1} \Delta Y_{i j}, \cdots, \sum_{i=1}^{n-1} \Delta Y_{i m}\right),
\end{aligned}
$$

where any value of factor $j$ shows the influence of factor $j$ on the change in the gross indicator $\Delta Y$. The total of all $\Delta Y_{j}(j=1,2, \cdots, m)$ constitutes the total gross of the gross indicator.

In this case, the contribution rate of the $j$ factor on economic growth is

$$
\frac{\Delta Y_{j}}{\Delta Y}, \quad j=1,2, \cdots, m
$$

Assume the constant elasticity of substitution production function model is

$$
\begin{aligned}
Y & =A\left(\delta_{1} K^{-\rho}+\delta_{2} L^{-\rho}+\delta_{3} E^{-\rho}+\delta_{4} C^{-\rho}+\delta_{5} I^{-\rho}\right)^{-\frac{\gamma}{\rho}} \\
& =A_{0} \mathrm{e}^{\lambda t}\left(\delta_{1} K^{-\rho}+\delta_{2} L^{-\rho}+\delta_{3} E^{-\rho}+\delta_{4} C^{-\rho}+\delta_{5} I^{-\rho}\right)^{-\frac{\gamma}{\rho}},
\end{aligned}
$$

where $A(t)=A_{0} \mathrm{e}^{\lambda t}$ is the technological progress level, $K$ is capital, $L$ is labor force, $E$ is energy consumption, $C$ is resident consumption, and $I$ is import and export. 
Assume $L_{i}$ is the cure connecting two points $M_{i}\left(Y_{i}, A_{i}, K_{i}, L_{i}, E_{i}, C_{i}, I_{i}\right)$ with $M_{i+1}\left(Y_{i+1}\right.$, $\left.A_{i+1}, K_{i+1}, L_{i+1}, E_{i+1}, C_{i+1}, I_{i+1}\right)(i=1,2, \cdots, n-1)$, which yields a curve equation that is

$$
\left\{\begin{array}{l}
Y_{t}=Y_{i}\left(\frac{Y_{i+1}}{Y_{i}}\right)^{t} \\
A_{t}=A_{i}\left(\frac{A_{i+1}}{A_{i}}\right)^{t} \\
K_{t}=K_{i}\left(\frac{K_{i+1}}{K_{i}}\right)^{t}, \\
L_{t}=L_{i}\left(\frac{L_{i+1}}{L_{i}}\right)^{t}, \quad 0 \leq t \leq 1 . \\
E_{t}=E_{i}\left(\frac{E_{i+1}}{E_{i}}\right)^{t} \\
C_{t}=C_{i}\left(\frac{C_{i+1}}{C_{i}}\right)^{t} \\
I_{t}=I_{i}\left(\frac{I_{i+1}}{I_{i}}\right)^{t}
\end{array}\right.
$$

In this case, the value of technological progress $A$ 's influence on $Y$ 's change in period $i$ is

$$
\begin{aligned}
\Delta Y_{i A}=\int_{L_{i}} \frac{\partial Y}{\partial A} \mathrm{~d} A & =\int_{L_{i}} \frac{Y}{A} \mathrm{~d} A \\
& =\int_{0}^{1} \frac{Y_{i}\left(\frac{Y_{i+1}}{Y_{i}}\right)^{t}}{A_{i}\left(\frac{A_{i+1}}{A_{i}}\right)^{t}} \cdot A_{i}\left(\frac{A_{i+1}}{A_{i}}\right)^{t} \cdot \ln \left(\frac{A_{i+1}}{A_{i}}\right) \mathrm{d} t \\
& =\int_{0}^{1} Y_{i}\left(\frac{Y_{i+1}}{Y_{i}}\right)^{t} \lambda \mathrm{d} t=\frac{\lambda\left(Y_{i+1}-Y_{i}\right)}{\ln \left(\frac{Y_{i+1}}{Y_{i}}\right)}
\end{aligned}
$$

The value of capital $K$ 's influence on $Y$ 's change in period $i$ is

$$
\begin{aligned}
& \Delta Y_{i K}=\int_{L_{i}} \frac{\partial Y}{\partial K} \mathrm{~d} K \\
& =\int_{L_{i}} A \frac{\gamma}{\rho}\left(\delta_{1} K^{-\rho}+\delta_{2} L^{-\rho}+\delta_{3} E^{-\rho}+\delta_{4} C^{-\rho}+\delta_{5} I^{-\rho}\right)^{-\frac{\gamma}{\rho}-1} \delta_{1} \rho K^{-\rho-1} \mathrm{~d} K \\
& =\int_{L_{i}} A \frac{\gamma}{\rho}\left(\frac{Y}{A}\right)^{\frac{\rho}{\gamma}\left(\frac{\gamma}{\rho}+1\right)} \delta_{1} \rho K^{-\rho-1} \mathrm{~d} K \\
& =\int_{0}^{1} A_{0} \mathrm{e}^{\lambda t} \frac{\gamma}{\rho}\left(\frac{Y_{i}\left(\frac{Y_{i+1}}{Y_{i}}\right)^{t}}{A_{0} \mathrm{e}^{\lambda t}}\right)^{1+\frac{\rho}{\gamma}} \delta_{1} \rho\left(K_{i}\left(\frac{K_{i+1}}{K_{i}}\right)^{t}\right)^{-\rho-1} K_{i}\left(\frac{K_{i+1}}{K_{i}}\right) \ln \frac{K_{i+1}}{K_{i}} \mathrm{~d} t \\
& =\int_{0}^{1} A_{0}^{-\frac{\rho}{\gamma}} \gamma \delta_{1} Y_{i}^{\left(1+\frac{\rho}{\gamma}\right)} K_{i}^{-\rho} \ln \frac{K_{i+1}}{K_{i}}\left[\mathrm{e}^{-\frac{\lambda \rho}{\gamma}}\left(\frac{Y_{i+1}}{Y_{i}}\right)^{1+\frac{\rho}{\gamma}}\left(\frac{K_{i+1}}{K_{i}}\right)^{-\rho}\right]^{t} \mathrm{~d} t \\
& =\frac{A_{0}^{-\frac{\rho}{\gamma}} \gamma \delta_{1} Y_{i}^{\left(1+\frac{\rho}{\gamma}\right)} K_{i}^{-\rho} \ln \frac{K_{i+1}}{K_{i}}}{\ln \left[\mathrm{e}^{-\frac{\lambda \rho}{\gamma}}\left(\frac{Y_{i+1}}{Y_{i}}\right)^{1+\frac{\rho}{\gamma}}\left(\frac{K_{i+1}}{K_{i}}\right)^{-\rho}\right]}\left[\mathrm{e}^{-\frac{\lambda \rho}{\gamma}}\left(\frac{Y_{i+1}}{Y_{i}}\right)^{1+\frac{\rho}{\gamma}}\left(\frac{K_{i+1}}{K_{i}}\right)^{-\rho}-1\right] \text {. }
\end{aligned}
$$


In the same way, the value of labor $L$ 's influence on $Y$ 's change in period $i$ is

$$
\begin{aligned}
\Delta Y_{i L} & =\int_{L_{i}} \frac{\partial Y}{\partial L} \mathrm{~d} L \\
& =\int_{L_{i}} A \frac{\gamma}{\rho}\left(\delta_{1} K^{-\rho}+\delta_{2} L^{-\rho}+\delta_{3} E^{-\rho}+\delta_{4} C^{-\rho}+\delta_{5} I^{-\rho}\right)^{-\frac{\gamma}{\rho}-1} \delta_{2} \rho L^{-\rho-1} \mathrm{~d} L \\
& =\int_{L_{i}} A \frac{\gamma}{\rho}\left(\frac{Y}{A}\right)^{\frac{\rho}{\gamma}\left(\frac{\gamma}{\rho}+1\right)} \delta_{2} \rho L^{-\rho-1} \mathrm{~d} L \\
& =\int_{0}^{1} A_{0} \mathrm{e}^{\lambda t} \frac{\gamma}{\rho}\left(\frac{Y_{i}\left(\frac{Y_{i+1}}{Y_{i}}\right)^{t}}{A_{0} \mathrm{e}^{\lambda t}}\right)^{1+\frac{\rho}{\gamma}} \delta_{2} \rho\left(L_{i}\left(\frac{L_{i+1}}{L_{i}}\right)^{t}\right)^{-\rho-1} L_{i}\left(\frac{L_{i+1}}{L_{i}}\right) \ln \frac{L_{i+1}}{L_{i}} \mathrm{~d} t \\
& =\int_{0}^{1} A_{0}^{-\frac{\rho}{\gamma}} \gamma \delta_{2} Y_{i}^{\left(1+\frac{\rho}{\gamma}\right)} L_{i}^{-\rho} \ln \frac{L_{i+1}}{L_{i}}\left[\mathrm{e}^{-\frac{\lambda \rho}{\gamma}}\left(\frac{Y_{i+1}}{Y_{i}}\right)^{1+\frac{\rho}{\gamma}}\left(\frac{L_{i+1}}{L_{i}}\right)^{-\rho}\right]^{t} \mathrm{~d} t \\
& =\frac{A_{0}^{-\frac{\rho}{\gamma}} \gamma \delta_{2} Y_{i}^{\left(1+\frac{\rho}{\gamma}\right)} L_{i}^{-\rho} \ln \frac{L_{i+1}}{L_{i}}}{\ln \left[\mathrm{e}^{-\frac{\lambda \rho}{\gamma}}\left(\frac{Y_{i+1}}{Y_{i}}\right)^{1+\frac{\rho}{\gamma}}\left(\frac{L_{i+1}}{L_{i}}\right)^{-\rho}\right]}\left[\mathrm{e}^{-\frac{\lambda \rho}{\gamma}}\left(\frac{Y_{i+1}}{Y_{i}}\right)^{1+\frac{\rho}{\gamma}}\left(\frac{L_{i+1}}{L_{i}}\right)^{-\rho}-1\right] .
\end{aligned}
$$

The value of energy $E$ 's influence on $Y$ 's change in period $i$ is

$$
\begin{aligned}
\Delta Y_{i E} & =\int_{L_{i}} \frac{\partial Y}{\partial E} \mathrm{~d} E \\
& =\int_{L_{i}} A \frac{\gamma}{\rho}\left(\delta_{1} K^{-\rho}+\delta_{2} L^{-\rho}+\delta_{3} E^{-\rho}+\delta_{4} C^{-\rho}+\delta_{5} I^{-\rho}\right)^{-\frac{\gamma}{\rho}-1} \delta_{3} \rho E^{-\rho-1} \mathrm{~d} E \\
& =\int_{L_{i}} A \frac{\gamma}{\rho}\left(\frac{Y}{A}\right)^{\frac{\rho}{\gamma}\left(\frac{\gamma}{\rho}+1\right)} \delta_{3} \rho E^{-\rho-1} \mathrm{~d} E \\
& =\int_{0}^{1} A_{0} \mathrm{e}^{\lambda t} \frac{\gamma}{\rho}\left(\frac{Y_{i}\left(\frac{Y_{i+1}}{Y_{i}}\right)^{t}}{A_{0} \mathrm{e}^{\lambda t}}\right)^{1+\frac{\rho}{\gamma}} \delta_{3} \rho\left(E_{i}\left(\frac{E_{i+1}}{E_{i}}\right)^{t}\right)^{-\rho-1} E_{i}\left(\frac{E_{i+1}}{E_{i}}\right) \ln \frac{E_{i+1}}{E_{i}} \mathrm{~d} t \\
& =\int_{0}^{1} A_{0}^{-\frac{\rho}{\gamma}} \gamma \delta_{3} Y_{i}^{\left(1+\frac{\rho}{\gamma}\right)} E_{i}^{-\rho} \ln \frac{E_{i+1}}{E_{i}}\left[\mathrm{e}^{-\frac{\lambda \rho}{\gamma}}\left(\frac{Y_{i+1}}{Y_{i}}\right)^{1+\frac{\rho}{\gamma}}\left(\frac{E_{i+1}}{E_{i}}\right)^{-\rho}\right]^{t} \mathrm{~d} t \\
& \left.=\frac{A_{0}^{-\frac{\rho}{\gamma}} \gamma \delta_{3} Y_{i}^{\left(1+\frac{\rho}{\gamma}\right)} E_{i}^{-\rho} \ln \frac{E_{i+1}}{E_{i}}}{\ln \left[\mathrm{e}^{-\frac{\lambda \rho}{\gamma}}\left(\frac{Y_{i+1}}{Y_{i}}\right)^{1+\frac{\rho}{\gamma}}\left(\frac{E_{i+1}}{E_{i}}\right)^{-\rho}\right]}\left[\frac{Y_{i+1}}{Y_{i}}\right)^{1+\frac{\rho}{\gamma}}\left(\frac{E_{i+1}}{E_{i}}\right)^{-\rho}-1\right] .
\end{aligned}
$$

The value of resident consumption $C$ 's influence on $Y$ 's change in period $i$ is

$$
\begin{aligned}
\Delta Y_{i C} & =\int_{L_{i}} \frac{\partial Y}{\partial C} \mathrm{~d} C \\
& =\int_{L_{i}} A \frac{\gamma}{\rho}\left(\delta_{1} K^{-\rho}+\delta_{2} L^{-\rho}+\delta_{3} E^{-\rho}+\delta_{4} C^{-\rho}+\delta_{5} I^{-\rho}\right)^{-\frac{\gamma}{\rho}-1} \delta_{4} \rho C^{-\rho-1} \mathrm{~d} C \\
& =\int_{L_{i}} A \frac{\gamma}{\rho}\left(\frac{Y}{A}\right)^{\frac{\rho}{\gamma}\left(\frac{\gamma}{\rho}+1\right)} \delta_{4} \rho C^{-\rho-1} \mathrm{~d} C \\
& =\int_{0}^{1} A_{0} \mathrm{e}^{\lambda t} \frac{\gamma}{\rho}\left(\frac{Y_{i}\left(\frac{Y_{i+1}}{Y_{i}}\right)^{t}}{A_{0} \mathrm{e}^{\lambda t}}\right)^{1+\frac{\rho}{\gamma}} \delta_{4} \rho\left(C_{i}\left(\frac{C_{i+1}}{C_{i}}\right)^{t}\right)^{-\rho-1} C_{i}\left(\frac{C_{i+1}}{C_{i}}\right) \ln \frac{C_{i+1}}{C_{i}} \mathrm{~d} t \\
& =\int_{0}^{1} A_{0}^{-\frac{\rho}{\gamma}} \gamma \delta_{4} Y_{i}^{\left(1+\frac{\rho}{\gamma}\right)} C_{i}^{-\rho} \ln \frac{C_{i+1}}{C_{i}}\left[\mathrm{e}^{-\frac{\lambda \rho}{\gamma}}\left(\frac{Y_{i+1}}{Y_{i}}\right)^{1+\frac{\rho}{\gamma}}\left(\frac{C_{i+1}}{C_{i}}\right)^{-\rho}\right]^{t} \mathrm{~d} t \\
& \left.=\frac{A_{0}^{-\frac{\rho}{\gamma}} \gamma \delta_{4} Y_{i}^{\left(1+\frac{\rho}{\gamma}\right)} C_{i}^{-\rho} \ln \frac{C_{i+1}}{C_{i}}}{\ln \left[\mathrm{e}^{-\frac{\lambda \rho}{\gamma}}\left(\frac{Y_{i+1}}{Y_{i}}\right)^{1+\frac{\rho}{\gamma}}\left(\frac{C_{i+1}}{C_{i}}\right)^{-\rho}\right]}\left[\frac{\mathrm{e}^{-\frac{\lambda \rho}{\gamma}}}{Y_{i}}\right)^{1+\frac{\rho}{\gamma}}\left(\frac{C_{i+1}}{C_{i}}\right)^{-\rho}-1\right] .
\end{aligned}
$$


The value of import and export I's influence on $Y$ 's change in period $i$ is

$$
\begin{aligned}
& \Delta Y_{i I}=\int_{L_{i}} \frac{\partial Y}{\partial I} \mathrm{~d} I \\
& =\int_{L_{i}} A \frac{\gamma}{\rho}\left(\delta_{1} K^{-\rho}+\delta_{2} L^{-\rho}+\delta_{3} E^{-\rho}+\delta_{4} C^{-\rho}+\delta_{5} I^{-\rho}\right)^{-\frac{\gamma}{\rho}-1} \delta_{5} \rho I^{-\rho-1} \mathrm{~d} I \\
& =\int_{L_{i}}^{L_{i}} A \frac{\gamma}{\rho}\left(\frac{Y}{A}\right)^{\frac{\rho}{\gamma}\left(\frac{\gamma}{\rho}+1\right)} \delta_{5} \rho I^{-\rho-1} \mathrm{~d} I \\
& =\int_{0}^{1} A_{0} \mathrm{e}^{\lambda t} \frac{\gamma}{\rho}\left(\frac{Y_{i}\left(\frac{Y_{i+1}}{Y_{i}}\right)^{t}}{A_{0} \mathrm{e}^{\lambda t}}\right)^{1+\frac{\rho}{\gamma}} \delta_{5} \rho\left(I_{i}\left(\frac{I_{i+1}}{I_{i}}\right)^{t}\right)^{-\rho-1} I_{i}\left(\frac{I_{i+1}}{I_{i}}\right) \ln \frac{I_{i+1}}{I_{i}} \mathrm{~d} t \\
& =\int_{0}^{1} A_{0}^{-\frac{\rho}{\gamma}} \gamma \delta_{5} Y_{i}^{\left(1+\frac{\rho}{\gamma}\right)} I_{i}^{-\rho} \ln \frac{I_{i+1}}{I_{i}}\left[\mathrm{e}^{-\frac{\lambda \rho}{\gamma}}\left(\frac{Y_{i+1}}{Y_{i}}\right)^{1+\frac{\rho}{\gamma}}\left(\frac{I_{i+1}}{I_{i}}\right)^{-\rho}\right]^{t} \mathrm{~d} t \\
& =\frac{A_{0}^{-\frac{\rho}{\gamma}} \gamma \delta_{5} Y_{i}^{\left(1+\frac{\rho}{\gamma}\right)} I_{i}^{-\rho} \ln \frac{I_{i+1}}{I_{i}}}{\ln \left[\mathrm{e}^{-\frac{\lambda \rho}{\gamma}}\left(\frac{Y_{i+1}}{Y_{i}}\right)^{1+\frac{\rho}{\gamma}}\left(\frac{I_{i+1}}{I_{i}}\right)^{-\rho}\right]}\left[\mathrm{e}^{-\frac{\lambda \rho}{\gamma}}\left(\frac{Y_{i+1}}{Y_{i}}\right)^{1+\frac{\rho}{\gamma}}\left(\frac{I_{i+1}}{I_{i}}\right)^{-\rho}-1\right] \text {. }
\end{aligned}
$$

Therefore, factor $A$ 's contribution rate to economic growth in periods $1 \sim n$ is

$$
\frac{\Delta Y_{A}}{\Delta Y}=\frac{\sum_{i=1}^{n-1} \Delta Y_{i A}}{\sum_{i=1}^{n-1} \Delta Y_{i A}+\sum_{i=1}^{n-1} \Delta Y_{i K}+\sum_{i=1}^{n-1} \Delta Y_{i L}+\sum_{i=1}^{n-1} \Delta Y_{i E}+\sum_{i=1}^{n-1} \Delta Y_{i C}+\sum_{i=1}^{n-1} \Delta Y_{i I}},
$$

factor $K$ 's contribution rate to economic growth in periods $1 \sim n$ is

$$
\frac{\Delta Y_{K}}{\Delta Y}=\frac{\sum_{i=1}^{n-1} \Delta Y_{i K}}{\sum_{i=1}^{n-1} \Delta Y_{i A}+\sum_{i=1}^{n-1} \Delta Y_{i K}+\sum_{i=1}^{n-1} \Delta Y_{i L}+\sum_{i=1}^{n-1} \Delta Y_{i E}+\sum_{i=1}^{n-1} \Delta Y_{i C}+\sum_{i=1}^{n-1} \Delta Y_{i I}},
$$

factor $L$ 's contribution rate to economic growth in periods $1 \sim n$ is

$$
\frac{\Delta Y_{L}}{\Delta Y}=\frac{\sum_{i=1}^{n-1} \Delta Y_{i L}}{\sum_{i=1}^{n-1} \Delta Y_{i A}+\sum_{i=1}^{n-1} \Delta Y_{i K}+\sum_{i=1}^{n-1} \Delta Y_{i L}+\sum_{i=1}^{n-1} \Delta Y_{i E}+\sum_{i=1}^{n-1} \Delta Y_{i C}+\sum_{i=1}^{n-1} \Delta Y_{i I}},
$$

factor $E$ 's contribution rate to economic growth in periods $1 \sim n$ is

$$
\frac{\Delta Y_{E}}{\Delta Y}=\frac{\sum_{i=1}^{n-1} \Delta Y_{i E}}{\sum_{i=1}^{n-1} \Delta Y_{i A}+\sum_{i=1}^{n-1} \Delta Y_{i K}+\sum_{i=1}^{n-1} \Delta Y_{i L}+\sum_{i=1}^{n-1} \Delta Y_{i E}+\sum_{i=1}^{n-1} \Delta Y_{i C}+\sum_{i=1}^{n-1} \Delta Y_{i I}},
$$

factor $C$ 's contribution rate to economic growth in periods $1 \sim n$ is

$$
\frac{\Delta Y_{C}}{\Delta Y}=\frac{\sum_{i=1}^{n-1} \Delta Y_{i C}}{\sum_{i=1}^{n-1} \Delta Y_{i A}+\sum_{i=1}^{n-1} \Delta Y_{i K}+\sum_{i=1}^{n-1} \Delta Y_{i L}+\sum_{i=1}^{n-1} \Delta Y_{i E}+\sum_{i=1}^{n-1} \Delta Y_{i C}+\sum_{i=1}^{n-1} \Delta Y_{i I}},
$$


and factor I's contribution rate to economic growth in periods $1 \sim n$ is

$$
\frac{\Delta Y_{I}}{\Delta Y}=\frac{\sum_{i=1}^{n-1} \Delta Y_{i I}}{\sum_{i=1}^{n-1} \Delta Y_{i A}+\sum_{i=1}^{n-1} \Delta Y_{i K}+\sum_{i=1}^{n-1} \Delta Y_{i L}+\sum_{i=1}^{n-1} \Delta Y_{i E}+\sum_{i=1}^{n-1} \Delta Y_{i C}+\sum_{i=1}^{n-1} \Delta Y_{i I}} .
$$

\section{Empirical Analysis of the Contribution Rates of Economic Growth Factors}

To further study tax revenue growth in China, we explore growth patterns and calculate the contribution rates of various input factors to tax revenue growth. The tax revenue $(Y)$ (unit: RMB 100 yuan) is selected as the comprehensive indicator of economic development, and the following economic influence factors are selected for the analysis: Fixed-asset investment $(K)$ (unit: RMB 100 million yuan); the number of employed people $(L)$ (unit: 10,000); energy consumption $(E)$ (unit: 10,000 tons of standard coal); resident consumption $(C)$ (unit: 100 million RMB); and import and export (I) (unit: RMB 100 yuan). See Table 1 for related data.

Table 1 Information of China's economic growth

\begin{tabular}{ccccccc}
\hline Year & $Y$ & $K$ & $L$ & $E$ & $C$ & $I$ \\
\hline 1994 & 5126.88 & 17042.1 & 67455.0 & 122737 & 18622.9 & 20381.9 \\
1995 & 6038.04 & 20019.3 & 68065.0 & 131176 & 23613.8 & 23499.9 \\
1996 & 6909.82 & 22913.5 & 68950.0 & 138948 & 28360.2 & 24133.8 \\
1997 & 8234.04 & 24941.1 & 69820.0 & 137798 & 31252.9 & 26849.7 \\
1998 & 9262.80 & 28406.2 & 70637.0 & 132214 & 33378.1 & 26967.2 \\
1999 & 10682.58 & 29854.7 & 71394.0 & 133831 & 35647.9 & 29896.2 \\
2000 & 12581.51 & 32917.7 & 72085.0 & 138553 & 39105.7 & 39273.2 \\
2001 & 15301.38 & 37213.5 & 72797.0 & 143199 & 43055.4 & 42183.6 \\
2002 & 17636.45 & 43499.9 & 73280.0 & 151797 & 48135.9 & 51378.2 \\
2003 & 20017.31 & 55566.6 & 73736.0 & 174990 & 52516.3 & 70483.5 \\
2004 & 24165.68 & 70477.4 & 74264.0 & 203227 & 59501.0 & 95539.1 \\
2005 & 28778.54 & 88773.6 & 74647.0 & 224682 & 68352.6 & 116921.8 \\
2006 & 34804.35 & 109998.2 & 74978.0 & 246270 & 79145.2 & 140974.0 \\
2007 & 45621.97 & 137323.9 & 75321.0 & 265480 & 93571.6 & 150648.1 \\
2008 & 54223.79 & 172828.4 & 75564.0 & 291448 & 114830.1 & 166863.7 \\
2009 & 59521.59 & 224598.8 & 75828.0 & 306647 & 132678.4 & 179921.5 \\
2010 & 73210.79 & 251683.8 & 76105.0 & 324939 & 156998.4 & 201722.2 \\
2011 & 89738.39 & 311485.1 & 76420.0 & 348002 & 183918.6 & 236402.0 \\
2012 & 100614.28 & 374694.7 & 76704.0 & 361732 & 210307.0 & 244160.2 \\
2013 & 110497.00 & 447074.0 & 76977.0 & 375252 & 237810.0 & 258267.0 \\
\hline & & & & & &
\end{tabular}


Assume the production function model is

$$
\begin{aligned}
Y & =F(A, K, L, E, C, I) \\
& =A(t)\left(\delta_{1} K^{-\rho}+\delta_{2} L^{-\rho}+\delta_{3} E^{-\rho}+\delta_{4} C^{-\rho}+\delta_{5} I^{-\rho}\right)^{-\frac{\gamma}{\rho}} \\
& =A_{0} \mathrm{e}^{\lambda t}\left(\delta_{1} K^{-\rho}+\delta_{2} L^{-\rho}+\delta_{3} E^{-\rho}+\delta_{4} C^{-\rho}+\delta_{5} I^{-\rho}\right)^{-\frac{\gamma}{\rho}} .
\end{aligned}
$$

Use the method proposed in the paper for parameter estimation and choose

$$
\eta^{(0)}=(0.01,0.01,0.1,0.1,0.1,0.1,0.1,0.1,0.1)
$$

as the initial value, assume a termination condition of $\left\|\delta^{(k)}\right\|<10^{-5}$; then

$$
\begin{aligned}
\eta & =\left(A_{0}, \lambda, \delta_{1}, \rho, \delta_{2}, \delta_{3}, \delta_{4}, \delta_{5}, \gamma\right) \\
& =(0.8220,0.0326,0.1820,0.0759,0.1802,0.1627,0.0412,0.0316,0.2869) .
\end{aligned}
$$

In other words, the production function model is

$$
\begin{aligned}
Y= & 0.8220 \mathrm{e}^{0.0326 t}\left(0.1820 K^{-0.0759}+0.1802 L^{-0.0759}+0.1627 E^{-0.0759}\right. \\
& \left.+0.0412 C^{-0.0759}+0.0316 I^{-0.0759}\right)^{-\frac{0.2869}{0.0759}} .
\end{aligned}
$$

The model's coefficient of determination is

$$
R^{2}=1-\frac{\sum\left(Y_{t}-\hat{Y}_{t}\right)^{2}}{\sum\left(Y_{t}-\bar{Y}\right)^{2}}=0.9947
$$

To compare the improved nonlinear regression algorithm in this paper with the conventional quasi-Newton and trust region methods in terms of precision and rate of convergence, the algorithm results are calculated using the parameters outlined above. Table 2 shows the results.

Table 2 Comparison of results of different algorithms

\begin{tabular}{cccc}
\hline Method & $\begin{array}{c}\text { Conventional } \\
\text { Quasi-Newton method }\end{array}$ & $\begin{array}{c}\text { Conventional Trust } \\
\text { Region method }\end{array}$ & $\begin{array}{c}\text { Improved nonlinear } \\
\text { regression algorithm }\end{array}$ \\
\hline$A_{0}$ & 0.4717 & 1.4541 & 0.8220 \\
$\lambda$ & 0.0726 & 0.2242 & 0.0326 \\
$\delta_{1}$ & 0.0820 & 0.0805 & 0.1820 \\
$\rho$ & 0.0359 & 0.0503 & 0.0759 \\
$\delta_{2}$ & 0.0802 & 0.2886 & 0.1802 \\
$\delta_{3}$ & 0.0827 & 0.0814 & 0.1627 \\
$\delta_{4}$ & 0.0812 & 0.0799 & 0.0412 \\
$\delta_{5}$ & 0.0816 & 0.0802 & 0.0316 \\
$\gamma$ & 0.2871 & 0.3183 & 0.2869 \\
\hline Number of iterations & 83 & 131 & 36 \\
Model's coefficient & 0.8942 & 0.9082 & 0.9947 \\
of determination $R^{2}$ & & & $1.1437 \mathrm{e}+08$ \\
Evaluation function & $2.2810 \mathrm{e}+09$ & $1.9782 \mathrm{e}+09$ & \\
$G(\eta)$ & & & \\
\hline
\end{tabular}


The improved nonlinear regression algorithm has fewer iterations than the conventional methods, the model has a high coefficient of determination, and the evaluation function has small value. Therefore, the model has a high fitting precision.

Then, we can observe that in 1994-2013, the contribution rate of technological progress is

$$
\frac{\Delta Y_{A}}{\Delta Y}=0.2082=20.82 \%
$$

the contribution rate of capital is

$$
\frac{\Delta Y_{K}}{\Delta Y}=0.4584=45.84 \%
$$

the contribution rate of labor is

$$
\frac{\Delta Y_{L}}{\Delta Y}=0.0422=4.22 \%,
$$

the contribution rate of energy is

$$
\frac{\Delta Y_{E}}{\Delta Y}=0.1351=13.51 \%
$$

the contribution rate of resident consumption is

$$
\frac{\Delta Y_{C}}{\Delta Y}=0.0873=9.73 \%,
$$

and the contribution rate of import and export is

$$
\frac{\Delta Y_{I}}{\Delta Y}=0.0488=5.88 \% \text {. }
$$

This demonstrates that in 1994-2013, the contribution rates of these factors to Chinese tax revenue growth are as follows: technological progress $-20.82 \%$; capital $-45.84 \%$; labor $-4.22 \%$; energy-13.51\%; resident consumption-9.73\%; and import and export-5.88\%. Chinese revenue growth depends on capital input first, technological progress second, energy third, resident consumption fourth, import and export fifth, and labor force last. This result agrees with China's actual situation.

\section{Conclusion}

This paper proposes a generalized form of the constant elasticity of substitution production function model, which is applicable to practical situations. As to the parameter estimation of the model, the paper proposes a nonlinear regression method with high precision and a fast rate of convergence. Also, this paper deduces a calculation formula to accurately calculate the economic growth contribution rate of various factors. This paper aims to promote the widespread use of the production function model and scientific calculation. The final section of this paper offers an empirical analysis of the contribution rates of Chinese economic growth factors, which will hopefully contribute to the economic planning and scientific decision-making of the government. 


\section{References}

[1] Cheng M L. China forecasting model of economic growth based on production function. Statistics and Decision, 2010, 20: 34-36.

[2] Li Z N, Pang W Q. Econometrics. Beijing: Higher Education Press, 2010.

[3] Sun J S, Ma S Q. Econometrics. Beijing: Tsinghua University Press, 2004.

[4] Hang B L, Wang W R, Ding L Q. An expand application to the CES production function. Quantitative \& Technica Economics, 1997, 8: 52-55.

[5] Kemfert C. Estimated substitution elasticities of a nested CES production function approach for Germany. Energy Economics, 1998, 20: 249-264.

[6] Kmenta J. On estimation of CES production function. International Economic Review, 1997, 8: 180-189.

[7] Avvakumov S N, Kiselev Yu N, Orlov M V, et al. Profit maximization problem for Cobb-Douglas and CES production functions. Computational Mathematics and Modeling, 2010, 21(3): 336-378.

[8] Pollard D, Radchenko P. Nonlinear least-squares estimation. Journal of Multivariate Analysis, 2006, 97(2): $548-562$.

[9] Kobayashi M, Narushima Y, Yabe H. Nonlinear conjugate gradient methods with structured secant condition for nonlinear least squares problems. Journal of Computational and Applied Mathematics, 2010, 234(2): $375-397$.

[10] Liu H L. A trust region algorithm for nonlinear least squares problems. Mathematics in Economics, 2007, 24(2): 213-216.

[11] Wang L Q, Leblanc A. Second-order nonlinear least squares estimation. Annals of the Institute of Statistical Mathematics, 2008, 60(4): 883-900.

[12] Cheng M L. A new measure model of factor contribution ratio for economic growth. Mathematics in Economics, 2004, 21(3): 235-239.

[13] Cheng M L. Correction and empirical analysis of the CES production function model. Journal of Engineering Mathematics, 2013, 30(4): 535-544.

[14] Cheng M L, Han Y. The measure model and analysis of contribution ratio of economic growth factor on Suzhou foreign capital manufacturing. Application of Statistics and Management, 2009, 28(3): 381-385.

[15] Yan M, Wang W Q. Estimating the contribution rate of education investment in the economic growth based on time-varying parameter. Statistics \& Information Forum, 2009, 24(7): 72-78.

[16] Zhou S S, Hu D L. Research on contribution rate of science and technology progress to economy growth. China Soft Science, 2010, 2: 34-39.

[17] Zhu Q. The influence of R\&D investment to economy growth. Scientific Management Research, 2009, 27(5): 95-110. 